\title{
QUALITY ASSESSMENT OF COMBINED IMU/GNSS DATA FOR DIRECT GEOREFERENCING IN THE CONTEXT OF UAV-BASED MAPPING
}

\author{
C. Stöcker ${ }^{a *}, F . N^{a}{ }^{a}$, M. Koeva ${ }^{a}$, M. Gerke ${ }^{b}$ \\ ${ }^{a}$ Faculty of Geo-Information Science and Earth Observation, University of Twente, Enschede, The Netherlands - (e.c.stocker, f.nex, \\ m.n.koeva)@utwente.nl \\ ${ }^{\mathrm{b}}$ Institute of Geodesy and Photogrammetry, Technische Universität Braunschweig, Germany - m.gerke@tu-bs.de
}

\section{ICWG I/II}

KEY WORDS: UAV, direct georeferencing, inertial measurement unit, exterior orientation optimization

\begin{abstract}
:
Within the past years, the development of high-quality Inertial Measurement Units (IMU) and GNSS technology and dedicated RTK (Real Time Kinematic) and PPK (Post-Processing Kinematic) solutions for UAVs promise accurate measurements of the exterior orientation (EO) parameters which allow to georeference the images. Whereas the positive impact of known precise GNSS coordinates of camera positions is already well studied, the influence of the angular observations have not been studied in depth so far. Challenges include accuracies of GNSS/IMU observations, excessive angular motion and time synchronization problems during the flight. Thus, this study assesses the final geometric accuracy using direct georeferencing with high-quality post-processed IMU/GNSS and PPK corrections. A comparison of different data processing scenarios including indirect georeferencing, integrated solutions as well as direct georeferencing provides guidance on the workability of UAV mapping approaches that require a high level of positional accuracy. In the current research the results show, that the use of the post-processed APX-15 GNSS and IMU data was particularly beneficial to enhance the image orientation quality. Horizontal accuracies within the pixel level $(2.8 \mathrm{~cm})$ could be achieved. However, it was also shown, that the angular EO parameters are still too inaccurate to be assigned with a high weight during the image orientation process. Furthermore, detailed investigations of the EO parameters unveil that systematic sensor misalignments and offsets of the image block can be reduced by the introduction of four GCPs. In this regard, the use of PPK corrections reduces the time consuming field work to measure high quantities of GCPs and makes large-scale UAV mapping a more feasible solution for practitioners that require high geometric accuracies.
\end{abstract}

\section{INTRODUCTION}

The advent of low cost, reliable, user-friendly and lightweight unmanned aerial vehicles (UAVs) have created new opportunities for collecting timely, tailored, detailed and highquality geospatial information (Colomina \& Molina 2014). However, time-consuming and costly ground truthing strategies - amongst others - are one aspect currently hindering large-scale UAV mapping projects that require high positional accuracies. Within the past years, the development of high-quality Inertial Measurement Units (IMU) and GNSS technology and dedicated RTK (Real Time Kinematic) and PPK (Post-Processing Kinematic) solutions for UAVs promise accurate measurements of the exterior orientation (EO) parameters which allow to georeference the images. The utilization of such devices has the ability to increasingly minimize or even eliminate the need to collect ground reference points. Subsequently, the derivation of large-scale UAV mapping outputs such as orthomosaics, digital surface models and $3 \mathrm{D}$ point clouds can become practical and economically feasible for various applications.

\subsection{Background}

Different studies already investigated systems and geometric accuracies achieved by the integration of sensor orientation parameters into UAV data processing workflows (Rehak \& Skaloud 2016; Gerke \& Przybilla 2016; Turner et al. 2014; Chiang et al. 2012; Haala et al. 2011). In this context, there is a general consensus that final positional accuracies are particularly influenced by the quality of the on-board sensors. The integration of projection centre coordinates obtained by low-cost IMU devices is not able to improve the quality of final results (Jóźków \& Toth 2014; Pfeifer et al. 2012), whereas projection centre coordinates obtained by high-quality GNSS instruments with RTK corrections from the ground can compete with geometric accuracies achieved by means of aerial triangulation and well distributed ground control points (GCPs) (Gerke \& Przybilla 2016; Mian et al. 2015; Rehak \& Skaloud 2016). In a study performed by (Gerke \& Przybilla 2016) the block orientation accuracy could significantly be enhanced by using an on-board RTK-GNSS solution. With an enabled RTK-GNSS and crossflight pattern, the best scenario reached a final horizontal geometric accuracy of $4 \mathrm{~cm}$. Interestingly, (Rehak \& Skaloud 2016) conducted a similar study but used means of relative aerial position and attitude control and introduced relative positional/angular observations of successive images. At this, the authors were able to significantly reduce the number of required ground control points in UAV mapping projects with weak block geometry. Final data products showed $2.8-3.0 \mathrm{~cm}$ of horizontal and $5.3 \mathrm{~cm}$ of vertical accuracy (Rehak \& Skaloud 2016).

\subsection{Motivation and Aim}

Large-scale mapping projects are preferably conducted using fixed wing UAVs which in turn generate their own challenges for direct sensor orientation. This includes excessive angular motion during turns and unavoidable flight dynamics due to wind

\footnotetext{
Corresponding author
} 
turbulences. Consequences embrace differences in forward and side image overlap. Large-scale mapping applications often require either a long flight time or multiple flight missions in order to cover the whole area of interest. Thus, image datasets might be affected by different illumination conditions which have further consequences on the image quality. Furthermore, all images with fixed wing UAVs are captured with cruising speed and the image quality could be lowered by motion blur. Next to this, large areas of interest often involve different land uses whereof some might show less textures or ambiguous features. All these aspects imply difficulties for conventional aerial triangulation which have an effect on the final data quality and accuracies. Angular misalignments and time synchronization offsets between the various instruments refer to another wellknown challenge for direct georeferencing.

Focused on cadastral UAV mapping projects that require a high level of geometric accuracy this study explores the potentials of direct georeferencing with post-processed (PPK) IMU-GNSS data. Whereas the positive impact of known precise GNSS coordinates of camera positions is already well studied, the influence of the angular observations has not been studied in depth so far. Furthermore - other than with RTK corrected IMU datasets - PPK data offers the opportunity to research the improvements of the GNSS corrections and their effect on the final data quality. To this end, a comparison of different test scenarios including indirect georeferencing, integrated sensor orientation as well as direct georeferencing will provide guidance on the level of accuracy that can be reached with various ground truthing setups and image processing configurations. Since absolute geolocation accuracy is key to cadastral surveying, the outcomes will ascertain the feasibility of this UAV mapping approach for cadastral applications.

\section{EQUIPMENT AND DATA COLLECTION}

\subsection{Platform and payload}

For this study, we chose a fixed wing UAV that is specialized for large area mapping: the DelairTech DT 18 UAV with PPK option. In contrast to RTK-GNSS corrections, PPK systems do not require a permanent radio link to the ground control station and thus are favourable for data acquisition in complex terrain settings and beyond visual line of sight. The coupled IMU/GNSS solution is made of an APX-15 Applanix system that is directly linked to the camera and thus prevents systematic errors due to unsynchronized IMU logs and camera exposures. The optical sensor of the payload is specifically designed for this UAV and comprises an industrial grade $5 \mathrm{MP}$ RGB sensor that is characterized by a focal length of $12 \mathrm{~mm}$ and a large pixel pitch $(3.45 \mu \mathrm{m})$. This configuration allows short exposure times and thus mitigates blurred images due to forward motion. Since the camera is rigidly installed in the UAV frame, the lever arm offset was computed once in the lab of the manufacturer.

\subsection{Study area}

With an extend of $1.4 \mathrm{~km}^{2}$, the test site surrounds the monastery "Benediktinerabtei Gerleve" which is located close to Coesfeld in North Rhine-Westphalia/Germany. It is characterized by a slightly undulated terrain with an altitude range from $140 \mathrm{~m}$ to $200 \mathrm{~m}$ ASL. A large part can be categorized as agriculturally used area. However, buildings, roads and trees are also represented.

\subsection{Data collection}

Field data collection encompassed the UAV data acquisition flight and RTK-GNSS based measurements of 22 ground reference points. More than 2500 images were captured to cover the whole study area. The camera was triggered by a predefined time interval of $1.5 \mathrm{sec}$ which corresponds to $80 \%$ forward overlap at the normal cruising speed of $16 \mathrm{~m} / \mathrm{s}$. To ensure a stable image block and reliable photogrammetric processing, side overlap was set to $70 \%$. The flight was performed in a constant height of $100 \mathrm{~m}$ above surface level and a regular flight pattern leading to a ground sampling distance (GSD) of $2.8 \mathrm{~cm}$. Following the recommendations for slightly undulated terrain by (Gerke \& Przybilla 2016) a cross flight was completed at the end of the data acquisition (see Figure 1). For several processing scenarios data-logs of the APX-15 were post-processed with Applanix POSPac UAV software ${ }^{1}$. Correction data was provided by $1 \mathrm{sec}$ interval RINEX data of a virtually created reference station located in the centre of the test area. The maximal baseline between the UAV platform and the virtual reference station is $650 \mathrm{~m}$.

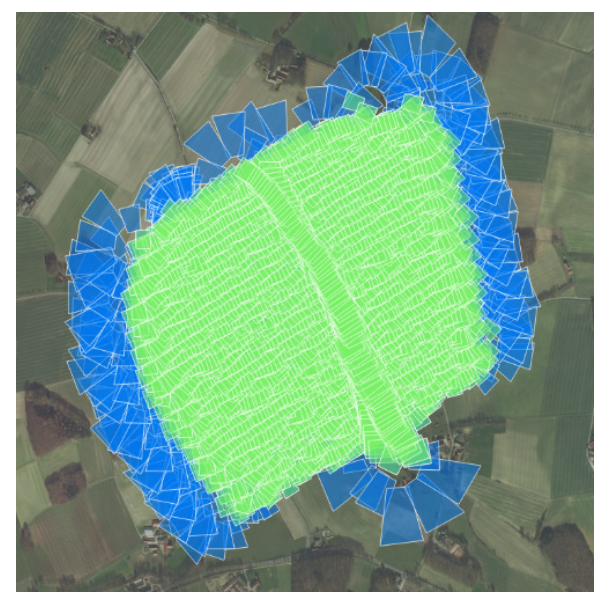

Figure 1: Flight pattern, green images are selected for photogrammetric processing

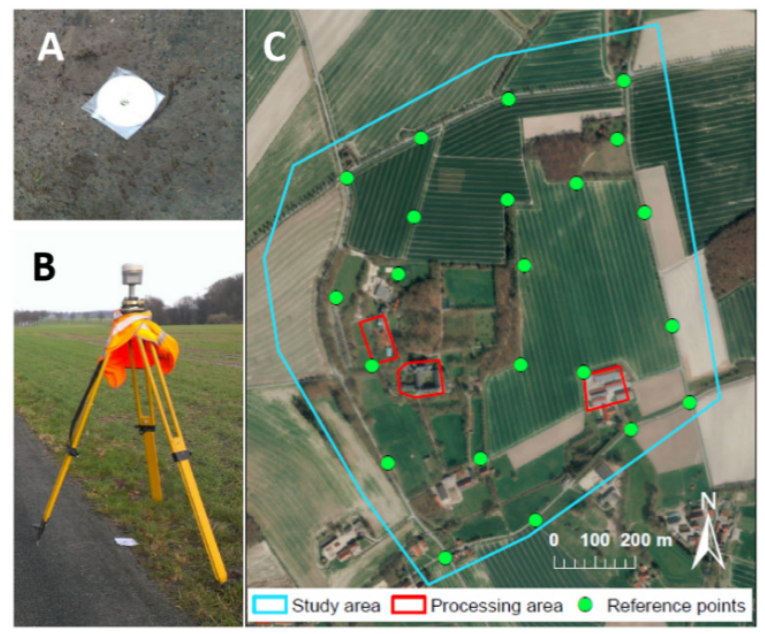

Figure 2: Ground reference points. A: shape, B: means of measurement, $\mathrm{C}$ : distribution of reference points and selected processing areas for point-to-plane comparison

\footnotetext{
${ }^{1} \mathrm{https}: / /$ www.applanix.com, accessed on June 262017
} 
As illustrated in Figure 2, ground reference points were made of white painted CDs with a diameter of $12 \mathrm{~cm}$. They were evenly distributed in the area of interest and fixed with a special surveying peg. The centre of the reference point was measured with a GNSS device on a tripod leading to survey accuracy of $2 \mathrm{~cm}$. Depending on the processed scenario, reference points were used either as GCPs or individual check points.

\section{METHODS}

\subsection{Image Processing}

The UAV image dataset is processed with the software Pix $4 \mathrm{D}^{2}$ and embraces eight different scenarios (S1-S8, see Table 1). The first two scenarios (S1 and S2) encompass indirect georeferencing, i.e. without using the GNSS observations on board the UAV and thus follow the classic photogrammetric approach that uses automatic aerial triangulation (AAT) and bundle block adjustment (BBA) to define the EO parameters. S1 and $\mathrm{S} 2$ are distinguished by their number of GCPs. The following four scenarios are characterized by integrated data processing using GCPs as well as IMU/GNSS information. Here, raw data of the APX-15 systems as well as PPK data is considered. Finally, the remaining two scenarios S7 and S8 follow the approach of direct georeferencing without using GCPs. In order to assess the quality of the calculated EO parameters, different weights were allocated. $\mathrm{X}, \mathrm{Y}$ and $\mathrm{Z}$ coordinates were assigned with a high weight for S3 - S8 whereas high and low weights for orientation parameter alter (cf. Table 1). Settings were adjusted within image orientation options of Pix4D. At the moment that GCPs are introduced (S3 - S6), the whole block geometry and thus also EO parameter are optimized. The weight for enabled GCPs (S1 - S6) was set to $3 \mathrm{~cm}$ in horizontal and $5 \mathrm{~cm}$ in vertical accuracy, respectively.

Table 1: Overview of scenarios for data processing

\begin{tabular}{|c|c|c|c|c|c|}
\hline Scenario & \multirow{2}{*}{$\begin{array}{c}\text { EO } \\
\text { data }\end{array}$} & \multicolumn{2}{|c|}{$\begin{array}{c}\text { EO parameters: } \\
\text { assigned weight for } \\
\text { image orientation }\end{array}$} & & \\
\cline { 3 - 5 } & & $\mathbf{X , Y , Z}$ & $\mathbf{\Omega , \Phi , K}$ & & \\
\hline S 1 & none & - & - & 18 & 4 \\
\hline S 2 & none & - & - & 4 & 18 \\
\hline S 3 & raw & high & low & 4 & 18 \\
\hline S 4 & raw & high & high & 4 & 18 \\
\hline S 5 & PPK & high & low & 4 & 18 \\
\hline S 6 & PPK & high & high & 4 & 18 \\
\hline S 7 & PPK & high & low & 0 & 22 \\
\hline S 8 & PPK & high & high & 0 & 22 \\
\hline
\end{tabular}

The UAV manufacturer delivered approximations for the interior orientations parameters that were adjusted during the data processing using a self-calibration.

\subsection{Data analysis}

Evidence about the final geometric accuracy and the overall performance is gained from several results: (1) check point residuals, (2) comparison of EO parameters for the various scenarios, and (3) point cloud characteristics using a point-toplane-based analysis.

\footnotetext{
${ }^{2}$ https://pix4d.com, accessed on June 262017
}

3.2.1 Check point residuals: The classical way to evaluate the geometric accuracy is the use of individual check points that are not taken into account during image processing. At this, the residuals are considered as difference between the observed values and the model. Here, check point coordinates serve as observed value and the calculated point position after photogrammetric processing as value in the model. Mean and standard deviation of check point residuals provide findings about the geometric accuracy and allow to detect systematic shifts and block deformations. Conditioned by the definition of different scenarios, the number of considered check points for this statistical evaluation varies.

3.2.2 Comparison of EO parameters: The second approach targets to explore the data processing performance of the various scenarios and encompass the comparison of EO parameters. Using this approach we can draw conclusions about positional uncertainties of projected points on the ground. The scenario with the lowest residuals at check point will serve as reference dataset. Differences of EO parameters were computed for the intersection of all images that were considered in all scenarios.

3.2.3 Point-to-plane based analysis: In addition to the classical approach to use check points to assess the orientation quality a dense image matching point cloud comparison is pursued. The approach follows the idea that if same dense matching techniques are applied for each BBA-configuration (same images, same settings, but just different input IO/EO parameters), the differences in quality of the resulting point clouds is just triggered by the particular IO/EO parameters. This allows to complete a relative accuracy check, even without reference. To this end we apply the method described by (Nex et al. 2015) to perform a point-to-plane-based analysis, resulting in signed point distances to planes, where we use the point cloud from the best BBA-configuration as reference. This enables to conclude on differences in systematic and random errors contained in the data.

\section{RESULTS}

\subsection{Data preparation - PPK option}

EO parameters for S5, S6, S7 and S8 were post-processed using the software tool POSPac UAV from Applanix. During this step the raw orientation were corrected with RINEX data of a virtual GNSS reference station in the centre of the study area. The postprocessed smoothed best estimated trajectory is shown in Fig. 3.

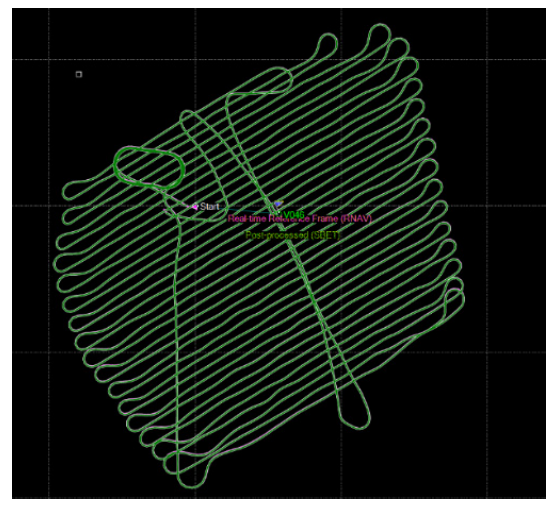

Figure 3: Representation of real-time reference frame (purple) and post-processed smoothed best estimated trajectory (green) 
After running the calculations, POSPac provides performance metrics that include the RMSE of positional/angular differences for the post-processed EO parameters for each second of the flight. Statistic of this performance metrics are outlined in Table 2 and deliver evidence of a clear improvement: uncertainties of positional parameters of more than one meter in the raw dataset were minimized to a few centimetre in the PPK dataset. The same applies to angular observations which were largely improved. Lowest accuracies were detected during turns where the IMU has difficulties to follow the change which in succession leads to higher uncertainties of IMU/GNSS observations than during smooth and straight parts of the flight route. Especially banked turns can block the view of the satellites from the GNSS antenna. However, maximum RMSE values for all positional observation are still below $3 \mathrm{~cm}$. This performance can be ascribed to the high quality of the APX-15 IMU instrument and the embedded Inertially-Aided Kinematic Ambiguity Resolution (IAKAR) technology (Hutton et al. 2008; Scherzinger \& Hutton n.d.).

Table 2: Performance metrics for real-time reference frame (raw) and post-processed smoothed best estimated trajectory (PPK)

\begin{tabular}{|l|c|c|c|c|}
\hline \multirow{2}{*}{} & \multicolumn{2}{|c|}{ Raw } & \multicolumn{2}{c|}{ PPK } \\
\cline { 2 - 5 } & Mean & Sigma & Mean & Sigma \\
\hline RMSE north [m] & 1.207 & 0.020 & 0.017 & 0.001 \\
\hline RMSE east [m] & 1.213 & 0.022 & 0.011 & 0.001 \\
\hline RMSE height [m] & 2.660 & 0.004 & 0.023 & 0.002 \\
\hline RMSE roll [arc min] & 9.023 & 1.346 & 2.398 & 0.557 \\
\hline RMSE pitch [arc min] & 8.855 & 1.118 & 2.606 & 0.583 \\
\hline RMSE yaw [arc min] & 30.214 & 4.381 & 9.904 & 2.340 \\
\hline
\end{tabular}

\subsection{Evaluation of check point residuals}

As shown in the Table 3 all scenarios are characterized by diverse statistics of the check point residuals.

Table 3: Mean and Sigma of check point residuals separated by scenarios

\begin{tabular}{|c|c|c|c|c|}
\hline & & $\mathbf{X}$ & $\mathbf{Y}$ & $\mathbf{Z}$ \\
\hline \multirow{2}{*}{ S1 } & Mean [m] & 0.084 & 0.269 & 1.512 \\
\cline { 2 - 5 } & Sigma [m] & 0.187 & 0.429 & 3.040 \\
\hline \multirow{2}{*}{ S2 } & Mean [m] & -0.242 & -0.055 & -9.284 \\
\cline { 2 - 5 } & Sigma [m] & 1.646 & 1.062 & 10.757 \\
\hline \multirow{2}{*}{ S3 } & Mean [m] & 0.124 & 0.042 & 0.107 \\
\cline { 2 - 5 } & Sigma [m] & 0.122 & 0.156 & 0.362 \\
\hline \multirow{2}{*}{ S4 } & Mean [m] & -0.075 & 1.072 & 0.696 \\
\cline { 2 - 5 } & Sigma [m] & 1.113 & 0.816 & 0.560 \\
\hline \multirow{2}{*}{ S5 } & Mean [m] & 0.001 & 0.008 & 0.033 \\
\cline { 2 - 5 } & Sigma [m] & 0.032 & 0.024 & 0.152 \\
\hline \multirow{2}{*}{ S6 } & Mean [m] & -0.757 & 0.571 & 0.492 \\
\cline { 2 - 5 } & Sigma [m] & 1.159 & 0.948 & 0.549 \\
\hline \multirow{2}{*}{ S7 } & Mean [m] & 0.217 & 0.186 & 0.053 \\
\cline { 2 - 5 } & Sigma [m] & 0.034 & 0.028 & 0.148 \\
\hline \multirow{2}{*}{ S8 } & Mean [m] & 0.156 & 0.502 & 0.727 \\
\cline { 2 - 5 } & Sigma [m] & 1.225 & 0.955 & 0.244 \\
\hline
\end{tabular}

Surprisingly, the conventional photogrammetric method with $\mathrm{AAT}$ and BBA in S1 does not deliver the expected geometric accuracy. Although 18 equally distributed GCPs were considered for the image processing, check point residuals for scenario are characterized by a comparatively high standard deviation. This can be attributed to difficulties during image matching due to low contrasts on uncultivated field plots and ambiguities at forest areas. Figure 4 exemplifies the small amount of automatic tie points that were found on areas with trees.
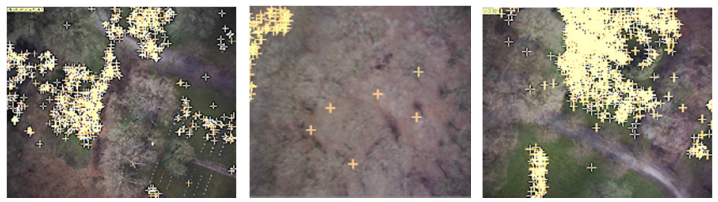

Figure 5: Examples for automatic tie points (crosses) on forest areas

In contrast to the remaining scenarios, S1 and S2 - those scenarios without initial EO parameters - present a significant difference of the horizontal and vertical accuracy values. This verifies the assumption that initial EO parameter approximations support tie-point extraction, accurate height reconstruction and finally avoid large block deformations.

The remaining six scenarios display a systematic pattern and one can clearly distinguish those scenarios with a high weight on angular observations and those with a lower weight. When positional as well as angular EO parameters are considered with a high weight (S4, S6, S8), significantly less tie-points can be found among matched images. For all three scenarios, the values for the standard deviation of the horizontal position is in the range of $1 \mathrm{~m}$. High mean values further evidence high block deformations. A close look to S4 and S6 embraces that both horizontal accuracies are at the same range even though $\mathrm{S} 4$ was calculated with raw EO parameters and S6 with more accurate PPK data. These results show, that the high weight of the angular observations affects the tie-point extraction and the image orientation negatively as it constraint the search of homologous points and furthermore the convergence towards minimized reprojection errors during the $\mathrm{BBA}$.

The results of S3, S5 and S7 - those scenarios where only positional EO parameters were assigned with a high weight and angular observations with a low weight - reveal higher geometric accuracies that go down to pixel level (GSD 2.8cm). In contrast to the statistical distribution of S4, S6 and S8, the PPK option shows large improvements of the block stability proven by low sigma values of S5 and S7. Furthermore, also the height component could be reconstructed more reliable with the PPK corrections. High mean and low sigma values for S7 indicate a systematic shift that can be explained by the absence of GCPs. S5 stands out for the lowest mean and sigma values and thus represents the scenario with the highest geometric accuracies and is therefore chosen as reference dataset (see 4.3 and 4.4.).

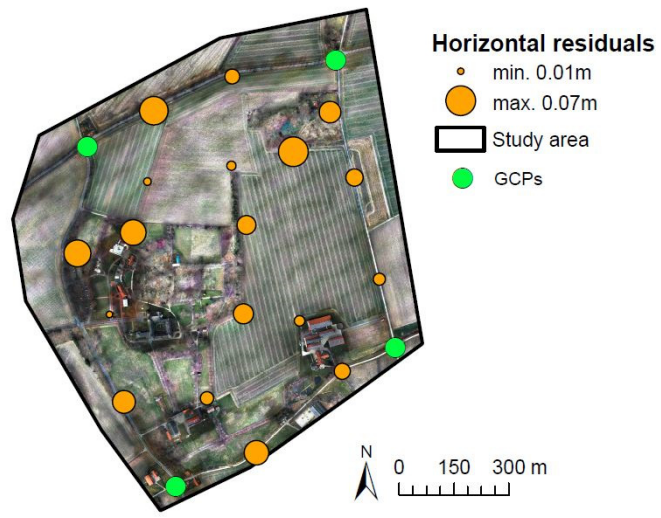

Figure 4: Horizontal residuals at check points for S5 
A detailed investigation of the check points in Figure 5 shows that lowest residuals are on open areas such as agricultural fields, buildings or infrastructure. Increasing residuals can be found in vicinity to trees or at margins of the study area. Here, ambiguous textures lead to poor results of the tie point extraction which in turn lower the block rigidity in these areas. The reprojection error of all 18 check points does not exceed 1 pixel $(\mathrm{RMSE}=0.5$ pixel).

\subsection{Comparison of EO parameter}

For this analysis, differences to reference dataset S5 were computed per image for all six EO parameter. Statistics of this relative quality measure are summarized in Table 4 and provide a first overview before the parameters are analysed in more detail.

Table 4: Mean and RMSE of EO parameter differences

\begin{tabular}{|c|c|c|c|c|c|c|c|}
\hline & & $\mathbf{x}[\mathbf{m}]$ & $\mathbf{y}[\mathbf{m}]$ & $\mathbf{z}[\mathbf{m}]$ & $\Omega\left[{ }^{\circ}\right]$ & $\Phi\left[{ }^{\circ}\right]$ & $\mathbf{K}\left[{ }^{\circ}\right]$ \\
\hline \multirow{2}{*}{ S1 } & Mean & 0.021 & 0.222 & \begin{tabular}{|l|}
-1.060 \\
\end{tabular} & -0.135 & 0.021 & 0.003 \\
\hline & RMSE & 2.735 & 2.029 & 4.765 & 1.263 & 1.665 & 0.167 \\
\hline \multirow{2}{*}{ S2 } & Mean & 0.078 & -2.601 & 2.899 & 1.626 & -0.475 & 0.214 \\
\hline & RMSE & 5.197 & 4.228 & 21.063 & 2.851 & 4.086 & 0.462 \\
\hline \multirow{2}{*}{ S3 } & Mean & -0.169 & 0.147 & 2.836 & -0.089 & -0.005 & -0.003 \\
\hline & RMSE & 0.352 & 0.434 & 2.875 & 0.247 & 0.170 & 0.075 \\
\hline \multirow{2}{*}{ S4 } & Mean & -0.071 & -0.503 & 0.110 & -0.175 & -0.166 & 0.061 \\
\hline & RMSE & 0.308 & 0.727 & 0.776 & 0.271 & 0.665 & 0.270 \\
\hline S5 & \multicolumn{7}{|c|}{ reference dataset } \\
\hline \multirow{2}{*}{ S6 } & Mean & 0.551 & -0.285 & 0.108 & -0.048 & -0.074 & 0.041 \\
\hline & RMSE & 0.627 & 0.422 & 0.383 & 0.194 & 0.663 & 0.102 \\
\hline \multirow{2}{*}{ S7 } & Mean & -0.208 & -0.171 & -0.053 & -0.004 & 0.006 & -0.001 \\
\hline & RMSE & 0.208 & 0.172 & 0.068 & 0.006 & 0.008 & 0.008 \\
\hline \multirow{2}{*}{ S8 } & Mean & -0.208 & -0.171 & -0.053 & -0.095 & -0.024 & 0.040 \\
\hline & RMSE & 0.208 & 0.172 & 0.068 & 0.211 & 0.660 & 0.102 \\
\hline
\end{tabular}

Comparable to the results of check point residuals, S1 and S2 stand out for their high RMSE values which are up to one decimal power higher than for the remaining scenarios. Compared to PPK option, raw data option of S3 and S4 shows higher RMSE values at the z-component which mirror the high uncertainties of the raw GNSS observables as shown in Table 2. Since no GCPs were introduced for $\mathrm{S} 7$ and $\mathrm{S} 8$, positional parameters $(\mathrm{x}, \mathrm{y}, \mathrm{z})$ were not optimized during BBA and remain the same for both scenarios. S7 demonstrates the lowest differences to the reference dataset.

As the EO parameters are not independent from IO parameter, Table 5 displays the focal length that was calculated during selfcalibration. Furthermore, the mean reprojection error provides a suitable measure to indicate the quality of the tie-points matching and the "tension" with external constraints (GCPs, GNSS and IMU information). In this regard, the high weight of the orientation parameters in S4, S6 and S8 does constrain the BBA and the self-calibration of the camera providing different focal lengths and higher re-projection errors.

Table 5: Focal length (f) and mean reprojection error (R) of BBA

\begin{tabular}{|c|c|c|c|c|c|c|c|c|}
\hline & S1 & S2 & S3 & S4 & S5 & S6 & S7 & S8 \\
\hline $\mathrm{f}[\mathrm{mm}]$ & 12.09 & 12.11 & 12.40 & 12.0 & 12.03 & 12.0 & 12.03 & 12.0 \\
\hline
\end{tabular}

\begin{tabular}{|l|l|l|l|l|l|l|l|l|}
\hline $\mathrm{R}$ [pixel] & 0.134 & 0.134 & 0.268 & 0.561 & 0.102 & 0.506 & 0.102 & 0.506 \\
\hline
\end{tabular}

The following two Figures reveal a detailed investigation of the variabilities of EO parameters during the entire image acquisition flight. Since the angular observations are in the main interest of this paper, only Omega and Phi were selected for a graphical representation. As evident from Table 3, S1 and S2 are in a different range and were not considered for the following comparison.

Differences in Omega and $P h i-$ as visualized in Figure 7 and 8 - show a similar systematic pattern for S4, S6 and S8 as the angular values provided by the IMU have a strong weight in the BBA and their values are almost the same.

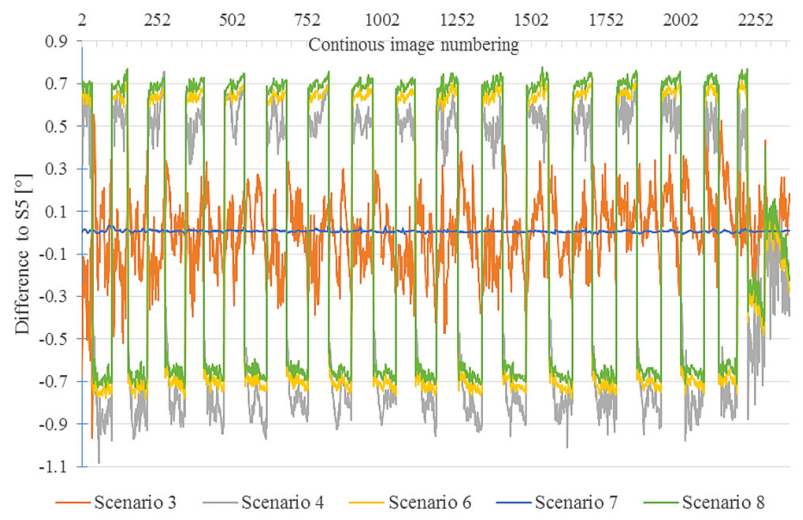

Figure 6: Differences in Omega

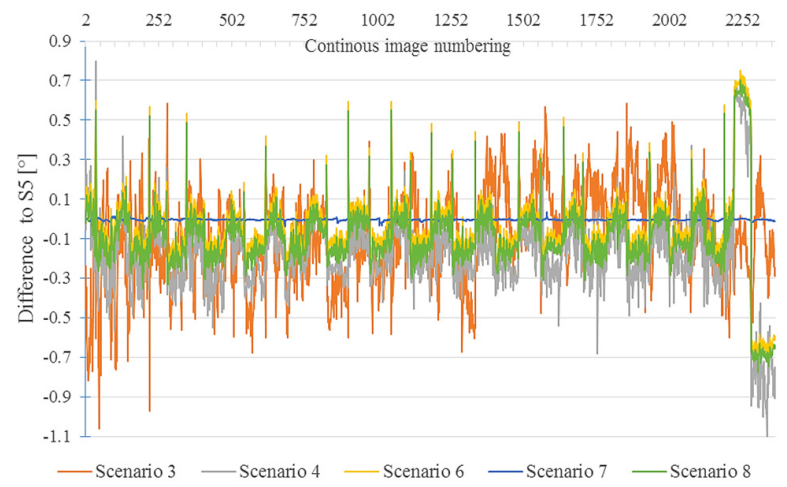

Figure 7: Differences in $P h i$

Positive and negative peaks for those scenarios change at each turn of the UAV and stay during straight lines with a constant offset of $+0.1 /-0.2^{\circ}$ for Omega and $+/-0.7^{\circ}$ for Phi. The systematism could be attributed to a small misalignment between the sensors which can be corrected when the angles parameters have less weight during image orientation process.

In contrast, differences for $\mathrm{S} 3$ do not show such a systematic pattern which can easily be correlated with the flight strips. For Phi observations, minimum and maximum peaks for S3 remain lower than peaks of S4, S6 and S8, respectively. In this case, the differences are mainly due to compensate the different information provided by the raw GNSS data. As expected from the RMSE values in Table 3, S7 displays almost identical angular orientation parameter.

\subsection{Point-to-plane analysis}

As described earlier, this analysis focus on the comparison of extracted planes. Since land uses such as meadows, forests and field are too noisy for the required planarity of this analysis, dedicated subareas with planar features such as roofs, paved roads and walkways were extracted. These areas include a farm, 
the monastery 'Benediktinerabtei Gerleve' and the building of the restaurant (Fig. 8).
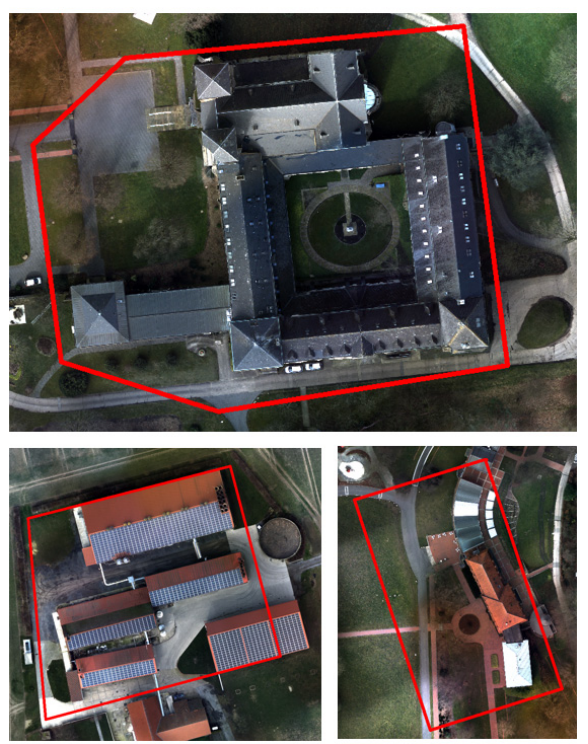

Figure 8: Selected areas for point-to-plane based comparison

Depending on the performance of the BBA the quality of dense matching outputs varied. Thus, not all selected planes showed sufficient point densities to be suitable for the point-to-plane analysis. Due to its high vertical offset S2 was no considered for this comparison as respective planes could not be assigned to each other.

The number of matching planes is shown in Table 6 and reveals insights into the data quality of reconstructed point clouds. S4, S6 and S8 show the smallest amount of matching planes. This result can be ascribed to the fact that predefined angular EO parameters are still too erroneous and limit the detection of homologous points during image orientation process. As an example, only half of all selected reference planes could be matched for S4.

Table 6: Number of matching planes that were included in the point-to-plane analysis

\begin{tabular}{|c|c|c|c|c|c|c|}
\hline & S1 & S3 & S4 & S6 & S7 & S8 \\
\hline Number of matching planes & 90 & 105 & 63 & 77 & 92 & 80 \\
\hline
\end{tabular}

As a recurrent phenomena in the results, extracted planes of S4, S6 and S8 show a high mean standard deviation for the point-toplane distances (see Fig. 9). This indicates a high level of noise and can be attributed to the poor quality of the input point clouds. In contrast, the results for S1 display a high systematic error (higher mean orthogonal distance than the mean standard deviation) that can be ascribed to the high positional offset (c.f. Table 2).

S3 shows the only positive value for the mean orthogonal distance and indicates a systematic shift. This can be explained by the fact that the EO parameters were based on raw observations that entail markedly higher positional uncertainties. S7 proves to be the scenario with the lowest positional offset although the value of the mean standard deviation indicate noise.

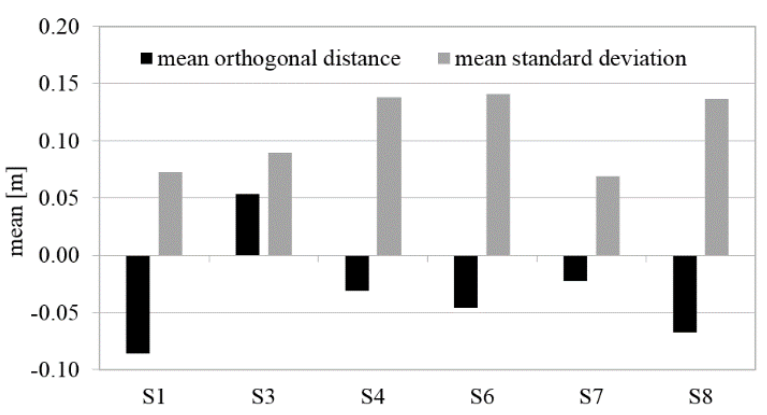

Figure 9: Statistics of signed point-to-plane distances separated by scenarios

\section{CONCLUSION}

During this study a UAV image dataset was processed with various ground truthing and georeferencing scenarios. Obtained accuracies were assessed by different methods including check point residuals, evaluation of EO parameters and point-to-plane distances. Particular focus was laid on the impact of different weights that were assigned of given EO parameters that included both - raw and PPK GNSS/IMU observations.

All results reveal clear evidence that known EO parameters (delivered by the on board sensors) are beneficial to guide the tiepoints matching, especially when the obtained UAV images impose challenges to the conventional AAT approach. This includes poorly textured areas, changing illumination conditions during the flight, and motion blur or image noise. Even with a dense network of GCPs, it was not possible to obtain the same level of accuracy as with use of raw EO parameter approximations. The use of the post-processed APX-15 GNSS and IMU data was particularly beneficial to enhance the data quality with pixel-level of horizontal accuracy. However, it was also shown, that the angular EO parameters are still too inaccurate to be assigned with a high weight during the image orientation process. Furthermore, detailed investigations of the EO parameters during the entire image acquisition flight unveil systematic sensor misalignments and offsets. This kind of errors could be easily fixed with a dedicated calibration of the lever arm of the system.

With highly accurate IMU/GNSS observations, the need of ground truthing can be reduced to a minimum of only 4 GCPs which were needed to avoid a systematic positional (mainly horizontal) offset of the dataset. Time consuming field work to measure high quantities of GCPs becomes obsolete and makes large-scale UAV mapping a more feasible solution for practitioners that require high geometric accuracies.

Further work will include more test flights with additional perpendicular strips and different flight heights to improve the self-calibration. Moreover, evident systematic errors in EO parameters will be analysed in more detail aiming to minimize respective error sources. Achieved accuracies with different GCPs setups will give a guideline to surveyors about the required number and distribution of GCPs for UAV flights in the context of cadastral mapping.

\section{ACKNOWLEDGEMENTS}

The research described in this paper was funded by the research project "its4land," which is part of the Horizon 2020 program of 
the European Union, project number 687828. The authors want to thank the monks of the monastery "Benediktinerabtei Gerleve" for their permission to conduct our data capture flights.

\section{REFERENCES}

Chiang, K.W., Tsai, M.L. \& Chu, C.H., 2012. The development of an UAV borne direct georeferenced photogrammetric platform for ground control point free applications. Sensors (Switzerland), 12(7), pp.9161-9180.

Colomina, I. \& Molina, P., 2014. Unmanned aerial systems for photogrammetry and remote sensing: A review. ISPRS Journal of Photogrammetry and Remote Sensing, 92, pp.79-97.

Gerke, M. \& Przybilla, H.J., 2016. Accuracy Analysis of Photogrammetric UAV Image Blocks: Influence of Onboard RTK-GNSS and Cross Flight Patterns. Photogrammetrie - Fernerkundung - Geoinformation, (12016), p.14.

Haala, N. et al., 2011. Performance test on UAV-based photogrammetric data collection. International Archives of Photogrammetry, Remote Sensing and Spatial Information Sciences, Vol. XXXVI(September), pp.1-6.

Hutton, J. et al., 2008. Tight Integration of GNSS Post-processed Virtual Reference Station with Inertial Data for Increased Accuracy and Productivity of Airborne Mapping. XXIst ISPRS Congress Technical Commission V, pp.3-11.

Jóźków, G. \& Toth, C., 2014. Georeferencing experiments with UAS imagery. ISPRS Annals of Photogrammetry, Remote Sensing and Spatial Information Sciences, II1(November), pp.25-29.

Mian, O. et al., 2015. Direct georeferencing on small unmanned aerial platforms for improved reliability and accuracy of mapping without the need for ground control points. International Archives of the Photogrammetry, Remote Sensing and Spatial Information Sciences - ISPRS Archives, 40(1W4), pp.397-402.

Nex, F. et al., 2015. ISPRS Benchmark for Multi-Platform Photogrammetry. Annals of Photogrammetry, Remote Sensing and Spatial Information Sciences, II3/W4(March), pp.25-27.

Pfeifer, N., Glira, P. \& Briese, C., 2012. Direct georeferencing with on board navigation components of light weight UAV platforms. International Archives of the Photogrammetry, Remote Sensing and Spatial Information Sciences - ISPRS Archives, 39(September), pp.487-492.

Rehak, M. \& Skaloud, J., 2016. Applicability of New Approaches of Sensor Orientation To Micro Aerial Vehicles. ISPRS Annals of Photogrammetry, Remote Sensing and Spatial Information Sciences, III-3(July), pp.441-447.

Scherzinger, B. \& Hutton, J., Applanix IN-Fusion TM Technology Explained. , pp.1-4. Available at: https://www.applanix.com/pdf/Applanix_IN-Fusion.pdf.

Turner, D., Lucieer, A. \& Wallace, L., 2014. Direct georeferencing of ultrahigh-resolution UAV imagery. IEEE Transactions on Geoscience and Remote Sensing, 52(5), pp.2738-2745. 